\title{
Autonomy and Morality in DRM and Anti-Circumvention Law
}

\author{
Dan L. Burk and Tarleton Gillespie
}

University of Minnesota Law School/Cornell University, Department of Communications

\begin{abstract}
Digital rights management technology, or DRM, provides self-enforcing technical exclusion from pre-determined uses of informational works. Such technical exclusion may supplement or even supplant intellectual property laws. The deployment of DRM has been subsidized by laws prohibiting both disabling of technical controls and assisting others to disable technical controls. To date the public debate over deployment of DRM, has been almost entirely dominated by utilitarian arguments regarding the social costs and benefits of this technology. In this paper, we examine the moral propriety of laws endorsing and encouraging the deployment of DRM. We argue that a deontological analysis, focusing on the autonomy of information users, deserves consideration. Because DRM shifts the determination of information use from
\end{abstract}

users to producers, users are denied the choice whether to engage in use or misuse of the technically protected work. State sponsorship of DRM in effect treats information users as moral incompetents, incapable of deciding the proper use of information products. This analysis militates in favor of legal penalties that recognize and encourage the exercise of autonomous choice, even by punishment of blameworthy choices, rather than the encouragement of technology that limits the autonomous choices of information users.

Keywords: intellectual property, DRM, digital rights management, copyright, autonomy, DMCA

\section{Introduction.}

The proliferation of personal computing technology at modest costs and the popular embrace of the Internet has resulted in widespread access to devices capable of unauthorized duplication and dissemination of creative informational works, and subsequently a legal, political, and societal scuffle over the application of copyright law to digital information. It has also prompted the deployment of an array of digital rights management (DRM) technologies, which aim to impose technical prohibitions on the unauthorized use of digital content by regulating access to that content. In this paper we explore the moral implications of this technology, arguing that the constraints imposed by DRM, and in particular by state sanctioned deployment of DRM, are detrimental to the respect that should be afforded to content users as autonomous individuals.

\section{DRM Technology.}

In its strongest form, DRM is intended to interdict unauthorized uses of protected content. Digitized works that have been technologically protected via DRM can only be accessed or used in accordance with the restrictions programmed into the devices by the owner of the work; other uses that might be preferred by recipients of the work become impossible. For example, a purchaser of music might wish to copy the music to a portable player, which the law permits; if the music is protected by DRM technologies, this will be impossible unless the music's copyright owner has programmed the DRM to allow such a copy. Such 
systems may also include monitoring or authentication features to track who is using what content and in what ways, in order to facilitate monetary charges or billing for access and use. Other DRM technologies, such as steganographic or "digital watermarking" technologies can identify and track the content they mark. (Godwin 2004)

In such a case of technological prohibition, absent the permission of the music owner, copies can only be made by disabling the DRM's technical protection, by hack or by crack. Of course, if technical protections can be disabled in order to allow legally permissible uses, they may be also disabled to allow impermissible copying or other improper use. Because of the possibility that they might be circumvented, DRM alone is unlikely to achieve the kind of protection desired by copyright owners; the DRM itself needs protection from circumvention via a state-sponsored legal regime that prevents users disabling the device. Since relatively few consumers have the technical skills to disable sophisticated DRMs, effective DRM protection also requires legal protection against those who might assist in circumvention, or provide tools to circumvent. Consequently, in the United States, the member states of the European Union, and many other nations, DRM has received the approval and imprimatur of the state in the form of anti-circumvention laws that prohibit users disabling DRM themselves or making available devices that might help others disable DRM.

\section{The Principles of the DRM Debate.}

Public debate over the propriety of DRM deployment has been based almost entirely upon utilitarian assessments of the efficacy, efficiency, and provisions of public goods. Intellectual property law generally has been seen as an economic incentive to promote the generation and distribution of creative works; by excluding unauthorized uses of a work, artificial scarcity is created and the owner of the work is assured the enviable market position of sole provider, providing them a way to reliably profit from the creation of such works. Intellectual property laws are said to maximize social welfare; and, if there are some costs to granting exclusive rights, they are justified by the greater benefits. (Landes and Posner 1989) To the extent that it merely supplements or extends such legal exclusion, technical protection in the form of DRM is viewed as a lower-cost, self enforcing adjunct to or substitute for intellectual property law, a means to the same worthy ends. (Stefik 1996)

Although such consequentialist arguments have been the primary justification for intellectual property, and so for DRM, deontological arguments have also been used on occasion to justify intellectual property laws. Deontological arguments focusing upon the author or producer of proprietary works are familiar in discussion of intellectual property rights and related public policies. Under this view, intellectual property rights are properly established by the state in order to recognize the autonomy and individuality of the author. The doctrine of "moral rights" in European copyright suggests that authors are seen to have a particular say over the presentation of their work that exceeds even the economic machinations of copyright law; it has been used to redress various artistic violations of cultural works, such as the colorization of classic films originally shot in black and white. This draws on the long tradition of using Locke's labor theory of property and Hegel's principle of will to justify copyright law -- not merely the utilitarian arrangement the U.S. Constitution describes, but a fundamental right to the work produced of your purposeful hand, as an extension of personality. (Drahos 1996; Hughes 1988) Such arguments might also be extended to DRM protection: DRM helps secure the rights of the creators of intellectual works, the fruits of their labor, extending their right to control the circulation and presentation of their work in contexts far from its point of origin.

Critics of DRM systems fret that this protection system is simultaneously a control system; they have challenged this system on a number of fronts. Some have challenged these technical protection measures as really a means to alter the purchase and use of cultural works to the economic benefit of the industries that produce them; while the fears of piracy with which they are justified may be reasonable ones, this copyright rhetoric is also a convenient distraction from the way DRM can regulate aspects of our interaction with culture that were never within the reach of copyright law. (Gillespie 2004; Gillespie, forthcoming) A number of critics have also challenged DRM for how its technical constraints may have 
consequences for the activities of users -- a worry about the autonomy of the user and their agency in the culture in which they are involved. Even DRM systems that only monitor or track content may have profound implications for individual autonomy. For example, Julie Cohen (1996) has shown how the monitoring features of content management technology are likely to "chill" certain types of reading, as consumers will be aware and perhaps apprehensive that their choices are being remotely observed and recorded. Such effects may be characterized as a subtle but pernicious curb on individual choice.

\section{The Problem of State-Sanctioned Preemption.}

In this paper we want to focus primarily on those technologies that are designed and deployed as "lock out" devices for content, to raise a more fundamental, and perhaps more philosophical, concern about the autonomy of the user. Most troublingly, these DRM technologies restrict the choice of the individual by fundamentally shifting the moment in which the use of information is regulated. Whereas legal prohibition leaves discretion over their behavior in the hands of the users, allowing them to determine whether to risk activity that might result in legal penalties, DRM forecloses such discretion, allowing only those actions determined in advance by the information producer. DRM works on the principle of preemption; while copyright law announces a set of rules and the consequences that may be applied to those who violate the rules, DRM builds these rules into the very tools with which we make any use at all, such that the prohibited uses are rendered impossible.

In some sense, this is a central part of DRM's appeal. For those who use DRM protections for their content, the fact that their rules will be automatically applied to everyone in every instance so as to keep copyright violations from ever occurring, is certainly preferable to a law that can only be applied after illicit copies have been made and distributed, the economic damage done and never to be undone. And the fact that these measures apply to all users equally - particularly since anti-circumvention laws will prohibit the technically literate from using their special skills for circumvention -- has a comforting sense of justice, more so than a law that applies only to those who get caught, and only when the copyright holder sees it as economically viable to bring suit.

However, there are three significant problems with a restriction that preempts behavior rather than applying consequences after the behavior has occurred. The first is that the kind of exceptions and special cases the law regularly accommodates may be rendered impossible. While we have a law prohibiting murder, and there is relatively little hesitation on either deontological or utilitarian grounds as to the merit of such a law, we have a series of caveats built into the law that we also value. Killing someone in selfdefense is still murder, but it is murder that, in the eyes of the law and after deliberation in a courtroom, can under particular circumstances be excused. Such an exception would cease to exist were the act of murder somehow preemptible in all cases.

Though we aspire to a legal regime that precisely and clearly regulates behavior in both morally and socially justifiable ways, we recognize that real-world contexts are always more complex than a statute can anticipate or accommodate; we rely on judges and juries to apply the abstractions of law to these messy cases, and to both honor the laws and to recognize those moments in which exceptions must be accommodated. Such discretion requires that the law be applied after the fact, though it means that crimes will be committed before they can be adjudicated. If the behavior has yet to occur, it cannot be judged, or can only be judged categorically and without consideration for individual circumstances.

This kind of concern has been the focus of some of the strongest critiques of DRM. Copyright as a legal doctrine attempts to strike a balance between the interests of authors, owners, and the public they serve. In the service of this balance, the otherwise exclusive rights granted to the owner of a copyrighted work are delimited by a set of important exceptions. Some of these exceptions are akin to self-defense in that they are legally sanctioned exceptions to the rule, rather than positive rules of their own. While the general rights of the copyright owner are violated, a judge may take into consideration the context and consequences of that violation, to determine whether the use fits a proscribed set of criteria protecting certain socially valuable unauthorized uses. Critics of DRM have noted that bright-line and technologically- 
enforced rules prohibiting duplication and distribution of copyrighted works renders impossible almost all such exemptions. (Burk and Cohen 2002; Felten 2003; Samuelson 2003) By preempting copying, DRM intervenes before use is made, meaning no unauthorized use can ever be made, permissible or otherwise.

\section{$5 \quad$ Preempting Rights of Conscience.}

Beyond the question of how a preemptive technological constraint handles exceptions and adjudications commonly managed in law, preemption also endangers the hallowed practice of protesting a law by breaking it. Just as we recognize that our laws are abstract and require constant adjudication, we also recognize that they are imperfect and require constant reconsideration. While we have mechanisms for making such changes, through legislation by our political representatives, we know this system often suffers from calcified unresponsiveness, bureaucratic inertia, and the powerful bias of moneyed interests. Often it is those citizens unfairly restricted by a rule who must rise up to challenge it; sometimes, the most viable means to do so is to break the rule as an act of political defiance, as civil rights protesters in the United States and South Africa historically did by willfully ignoring segregationist or apartheid laws. They did not presume that they would elude the consequences the law set out for their actions; they decided that the statement made by defying the law and the possibility of change were worth accepting those consequences.

Hackers who have challenged DRM restrictions have sometimes adopted a similar rationale, dubbing their circumventing activities a form of "electronic civil disobedience." The comparison to human rights agitation is not meant to overdramatize digital copyright law and its discontents, nor to suggest that all those who provided DeCSS had political critique in mind. It is merely to point out that the mechanism used by the protesters in both cases, defying the law to call attention to its immorality, is an important tactic for an imperfect democratic system. Preemption makes such critiques impossible -- except so long as hackers can circumvent these restrictions. Such circumventions will continue to be made, both for fun and as a political statement. But in the dream of DRM providers, such circumvention will eventually be preempted itself through more powerful encryption and more robustly designed technologies.

\section{Undermining Autonomy.}

Finally, beyond the possible restriction of justifiable exceptions and of civil disobedience, preemption raises a more fundamental issue, one that asks us to ponder the very justification of law itself and the right to impose restrictions on another person. In the context of criminal law, Herbert Morris (1968) notes that systems of state-sponsored deterrence encompass two different modes of behavioral intervention: the criminal and the medical. The former assumes that the perpetrator is capable of independent, autonomous choice, and so is responsible for his or her actions. Even though society may judge the actions to be blameworthy and morally culpable, criminal law assumes that they were freely chosen by the perpetrator and so deserving of punishment. Indeed, society in some sense pays the perpetrator a backhanded compliment by punishing blameworthy actions: in assigning responsibility and blame for the action, society affirms the moral status of the perpetrator as an autonomous individual.

In contrast to criminal punishment, the state may instead adopt a medical paradigm. The court can assume that the individual is not an autonomous actor, but is in some sense incompetent to make fully formed choices: that is, that the perpetrator is mentally or morally immature or incapacitated. The individual's anti-social behaviors are excused if the person is deemed unable to appreciate either the consequences of the action, or unable to understand the moral principle violated. This relieves the perpetrator of responsibility for his or her actions, but this comes at a price. The finding of diminished capacity or insanity typically results in forcible institutionalization, not as punishment, but for treatment of the disorder, as well as for the protection of the individual's well-being. Such a finding is also typically accompanied by wholesale revocation of the individual's legal rights, including rights of bodily integrity, allowing the state to engage in treatment of the disorder with or without the individual's consent. Because the individual is deemed incapable of making choices, the state or another individual appointed by the 
state assumes responsibility deserving of pity, but not deserving of recognition as a fully autonomous person.

Adopting a similar framework to that developed by Morris, we might view state sponsorship of DRM as a denial of the responsibility, and so of the autonomy, otherwise accorded to information users. DRM, as we have shown, shifts discretion away from information users, placing it in the hands of information producers. Because DRM preempts the discretion of information users, denying them the choice as to how information products are to be used, it treats them as morally immature or incompetent, preempted from, and by implication incapable of, making a moral decision to obey the law. DRM places information producers in the role of the parent, treating users as children, locking away the cookie jar because users are incapable of independently determining the responsible use of information products. Of course the individual may seek the DRM owner's consent in order to gain access to the content, or their approval of the user's desired use. But positioning the user's actions at the mercy of the DRM owner's consent only underscores the infantilization at work.

\section{Undermining the Legitimacy of Law.}

This preemption of responsibility can be challenged on consequentialist grounds as well. A traditional system of law -- in which particular behaviors are prohibited, and in which perpetrators proven to have violated the prohibition are duly punished -- establishes its legitimacy on at least two grounds. First, the code of appropriate behavior articulated by a legal system will enjoy a sense of legitimacy to the extent that it roughly matches an underlying and widely shared morality. While the particular prohibitions may not always be the most exact or fair or perfectly described, and the system by which they are enforced may be plagued by the frailties of human and institutional capacity, those subject to it may nevertheless accept it if it feels like a sufficiently close match to their own personal and communal sense of right and wrong. Second, in a democratic society, we expect that these laws have been written and enacted according to the general wishes of the public upon which they are to be imposed, at least by way of their elected officials. The process by which they are produced lends them legitimacy, as does the moral principles to which they adhere.

But perhaps there is a third element that also lends legitimacy to the rule of law, one we rarely need to consider because it is unavoidably true when the law is only capable of acting after a crime is committed. A citizen may only feel a remote sense that the system of law to which they are subject was chosen by them -- the chain of people and decisions that link that citizen to the sites in which laws are produced can feel long and flimsy. And they may not necessarily sense an ideal match between it and their own personal moral beliefs. But every time this citizen chooses to obey the law, they offer that system a tiny bit of legitimacy, both in their own mind and on a societal level. The very fact that a law can be broken, that I could pocket that candy bar if I so chose, means that I must in every moment choose not to. And with that palpable sense of autonomy, even in the face of laws I don't particularly believe in or feel are unlikely to be enforced, I grant the system of law legitimacy. And I am surrounded by others doing the same, a constant reminder of the legitimacy of these legal norms.

A preemptive restraint, such as DRM, evacuates this sense of agency. The average citizen cannot possibly break the law, and in fact may not even know exactly what this law allows and prohibits. Rather than being contemplated and accepted in every instance, DRM regulates by obviating that choice altogether. The legitimacy of its authority over people, then, cannot be built on the active consent of its subjects. Instead, DRM, by intervening before behavior and preempting possible uses, authorized and otherwise, instrumentalizes users as mechanisms designed to collectively pay the artist's salary, nothing more.

\section{Qualifying the Argument.}

To be certain, as our cookie jar analogy above indicates, there already exist other situations in which technology is used to block access to the use of property, and where the state encourages and sanctions 
the use of such technologies. Locks are commonly used to preempt access to physical property, and the state may prohibit the circumvention of such locks and the provision of lock-picking tools. Do our concerns about the preemptive character of DRM suggest that the very notion of locks and walls should be discarded?

First, it is worth noting when considering such examples that the law typically recognizes certain defenses or excuses for violation of the legal prohibition. For example, the law relieves an actor of culpability when an overriding need, typically for the preservation of life, is at stake. Unlike the disease model of behavior, such defenses do not relieve the actor of responsibility, but rather recognize an exception to the general rule in which the actor acted properly, if contrary to law. And to be fair, U.S. and European anti-circumvention law also allows such exceptions: for example, under the U.S. statute. libraries may circumvent DRM in order to assess content they are considering acquiring, legitimate software designers and computer scientists can circumvent DRM to reverse engineer the code, parents may circumvent DRM on filtering software to see what websites are being blocked. Critics have noted, however, that the near-total prohibition on circulating circumvention tools leaves most of these people without the practical means to enjoy these rights.

But deployment of DRM to exclude information users must be differentiated from state-sanctioned uses of technology to secure physical property, because information goods are uniquely necessary for the definition and development of the self and for participation in culture and the democratic process. U.S. copyright law is built on this central tension: rights to cultural expression are handed to individuals so that the work may be marketed as a commodity to ensure both its dissemination and the compensation of its creator, but this granted right is justifiable precisely because of the need, or right, of the public to freely and widely available culture and knowledge of all sorts. Despite the metaphor of property, the law often recognizes that culture is unlike material property in crucial ways. Information goods offer the cultural and intellectual sustenance that allows us to flourish as intellectual and political beings. Consequently, the user of an informational work is in a unique position to assess his or her need for access to it. Denying users the opportunity to make such an assessment not only risks denying their access to necessary informational works, but the opportunity to make an independent determination as to that need. Legal punishment both excuses the proper unauthorized use of informational works and punishes the culpable, but responsible, misuse of the works. DRM permits neither of these interventions.

\section{Conclusion.}

In its strongest form, DRM "lock-out" technology is morally troubling, due to the preemptive constraints it imposes upon information users. These constraints tend to undermine the moral legitimacy of democratic law, to prohibit the exercise of conscience disobedience against the law, and to treat as moral incompetents. Unlike technologies used to secure physical goods, these technological constraints occur in the context of cultural and informational content basic to human flourishing, a further insult to the autonomous choice of the individual. The legitimation of the technology by the state is additionally troubling if one believes, as we do, that the proper role of the state is to foster individual autonomy and flourishing. These factors militate in favor of careful re-consideration of the global rush to deployment of such technologies.

\section{References}

Burk, D. and Cohen, J. (2001). Fair Use Infrastructure for Copyright Management Systems, Harvard Journal of Law \& Technology, $15,41-83$.

Cohen, J. (1996). A Right to Read Anonymously, Connecticut Law Review 28, 981

Drahos, P. (1996). A Philosophy of Intellectual Property, Dartmouth, Brookfield.

Felten, E. (2003). A Skeptical View of DRM and Fair Use, Communications of the ACM, 46(4), 57-59.

Gillespie, T. (2004). Copyright and Commerce: The DMCA, Trusted systems, and the Stabilization of Distribution, The Information Society, 20(4), 239-254.

Gillespie, T. (forthcoming). Technology Rules: Copyright and the Realignment of Digital Culture, MIT Press, Cambridge, MA. 
Godwin, M. (2003). What Every Citizen Should Know about DRM, a.k.a. 'Digital Rights Management'. Public Knowledge. Washington D.C.: New America Foundation. Available at http://www.publicknowledge.org/pdf/citizens guide to drm.pdf; last accessed September 5, 2005.

Hughes, J. (1988). The Philosophy of Intellectual Property, Georgetown Law Review, 77, 287-291.

Landes, W.M. and Posner, R.A. (1989). An Economic Analysis of Copyright Law, Journal of Legal Studies 18, 325-363.

Lessig, L. (1999). Code and Other Laws of Cyberspace, Basic Books, New York.

Morris, H. (1968). Persons and Punishment. The Monist, 52(4), 475-501.

Samuelson, P. (2003). Digital Rights Management \{and, or, vs.\} the Law, Communications of the ACM, 46(4), 41-45.

Stefik, M. (1996). Letting loose the light: Igniting commerce in electronic publication, in Internet Dreams: Archetypes, Myths, and Metaphors, ed. Mark Stefik, MIT Press, Cambridge, MA, 219-254. 